THEOREM. If $f$ and $g$ are integral forms of unit determinant, then $f$ and $g$ are integrally equivalent if and only if (1) they have the same number of variables, (2) they represent the same numbers modulo $\pi^{2 e+1},(3) S(f)=$ $S(g)$, and (4) $d(f) / d(g)$ is a square.

\title{
THE AXIOM OF CHOICE IN QUINE'S NEW FOUNDATIOŃS FOR MATHEMATICAL LOGIC
}

\author{
By ERNST P. Specker \\ Eidgen. Technische Hochschule, Zurich \\ Communicated by $\mathrm{H}$. Weyl, July 3,1953
}

The object of this note is to disprove the axiom of choice in Quine's "New Foundations." 1 As the axiom of choice is provable for finite sets, we obtain the axiom of infinity as a corollary. ${ }^{2}$

There will be references to Rosser's Logic for Mathematicians, ${ }^{3}$ although the axiom of infinity is assumed there; but it is readily eliminated for our purposes if one replaces Quine's ordered pair by Kuratowski's.

The proof will be by reductio ad absurdum.

1.1 $V$ is the universal set, $\Lambda$ the null set (R 256).

$1.2 S C(a)$ is the set of subsets of a (R 255).

$1.3 S C(V)=V(\mathrm{R} 256)$.

$1.4 \operatorname{USC}(a)$ is the set of unit subsets of $a, \operatorname{USC}^{2}(a)=\operatorname{USC}(\operatorname{USC}(a))$ (R 255).

1.5 Fin is the set of finite sets ( $R$ 417).

2.1 Cardinal numbers are construed as saturated sets of equivalent sets (R 371). $N c(a)$ is the cardinal number of $a$; so $a \in N c(a)$.

$2.2 N C$ is the set of cardinal numbers; $\Lambda \notin N C$.

$2.3 N c(S C(a))=N c(S C(b))$ if $N c(a)=N c(b)(\mathrm{R} 369)$.

$2.4 N c(U S C(a))=N c(U S C(b))$ if and only if $N c(a)=N c(b)(\mathrm{R} 368)$.

$2.5 N c(S C(V))=N c(V)(1.3)$.

$2.6 N c(S C(U S C(a)))=N c(U S C(S C(a)))(\mathrm{R} 368)$.

$2.7 N c(S C(\operatorname{USC}(V)))=N c(U S C(V))$ (2.6 and 1.3).

2.8 A cardinal number is finite if it is a subset of Fin. FNC is the set of finite cardinal numbers.

$2.91,2,3$ are defined as cardinal numbers of sets with one, two, three elements; $1,2,3 \in F N C$.

3.1 Definition of the sum of two cardinal numbers $m, n: m+n=$ $N c(a \cup b)$ if $m=N c(a), n=N c(b)$ and $a n b=\Lambda$; if there are no such $a, b$, then $m+n=\Lambda$ (R 373).

$3.21+1=2,1+2=3$. 
3.3 If $n$ is a finite cardinal number and $n+1 \neq \Lambda$, then $n+1$ is a finite cardinal number.

3.4 If $m$ is a finite cardinal number, then there are finite cardinal numbers $n, p, q$ such that either $m=n+n+n$ or $m=p+p+p+1$ or $m=q+q+q+2$; either of these three cases excludes the two others.

3.5 The cardinal numbers are well-ordered by the relation "there are sets $a, b$ such that $a \in m, b \in n$ and $a \subseteq b$ " (axiom of choice).

3.6 If $n+1$ is a finite cardinal number, then $n<n+1$.

4.1 Definition of $2^{m}$ for cardinal numbers $m$ : If $m=N c(\operatorname{USC}(a))$, then $2^{m}=N c(S C(a))(\mathrm{cf} .2 .3,2.4 ; \mathrm{R} 389)$; if there is no set $a$ such that $U S C(a) \in m$, then $2^{m}=\Lambda$.

$4.22^{N c(U S C)(a))}=N c(S C(a))$.

$4.32^{N_{c}(U S C(V))}=N c(V)(4.2$ and 2.5$)$.

$4.42^{N c\left(U S C^{2}(V)\right)}=N c(U S C(V))(4.2$ and 2.7$)$.

$4.52^{m}=\Lambda$ if and only if $N c(\operatorname{USC}(V))<m$.

4.6 If $2^{m} \neq \Lambda$, then $m<2^{m}$ (R 390).

$4.7 N c(U S C(V))<N c(V)$ (4.3 and 4.6).

4.8 If $m \leq n$ and $2^{n} \neq \Lambda$, then $2^{m} \neq \Lambda$ and $2^{m} \leq 2^{n}$.

4.9 " $2^{m}=n$ " is stratified if " $m$ " and " $n$ " have the same type.

5.1 Definition of $T(m)$ for cardinal numbers $m: T(N c(a))=$ $N c(U S C(a))$ (cf. 2.4).

$5.2 T(1)=1, T(2)=2, T(N c(V))=N c(U S C(V)), T(N c(U S C(V)))=$ $N c\left(\operatorname{USC}^{2}(V)\right)$.

5.3 If $m, n, m+n$ are cardinal numbers, then $T(m+n)=T(m)+$ $T(n)$.

5.4 If $m$ is a finite cardinal number, then $m \neq T(m)+1$ and $m \neq$ $T(m)+2(3.4,5.2,5.3)$.

5.5 If $m, n$ are cardinal numbers, then $m \leq n$ if and only if $T(m) \leq$ $T(n)$ (2.4).

5.6 If $m \leq T(n)$, then there is a $p$ such that $m=T(p)$

5.7 If $m \leq N c(U S C(V))$, then there is a $p$ such that $m=T(p)$.

$5.82^{T(m)} \neq \Lambda(45)$

5.9 If $2^{m} \neq \Lambda$, then $T\left(2^{m}\right)=2^{T(m)}(2.6$ and 4.2$)$.

6.1 If $m$ is a cardinal number, then $\Phi(m)$ is the set of cardinal numbers $m, 2^{m}, 2^{2^{m}}, \ldots$ To formalize this, define $Q(m, n)$ for $m, n \in N C$ and $2^{m}=n ; \phi(m)=\operatorname{Clos}(\{m\}, Q)(\mathrm{R} 245 ;$ stratification 4.9$)$.

6.2 If $2^{m}=\Lambda$, then $\Phi(m)=\{m\}$.

$6.3 \phi(N c(V))=\{N c(V)\}, N c(\phi(N c(V)))=1$.

6.4 If $n \in \phi(m)$, then $m \leq n$.

6.5 If $2^{m} \neq \Lambda$, then $m \notin \phi\left(2^{m}\right)$ (4.6).

6.6 If $2^{m} \neq \Lambda$, then $\phi(m)=\{m\} \cup \Phi\left(2^{m}\right)$.

Proof: (1) $\phi(m) \subseteq\{m\} \cup \phi\left(2^{m}\right): m \in\{m\} \cup \phi\left(2^{m}\right)$; assume $n \in\{m\} \cup$ $\phi\left(2^{m}\right)$ and $2^{n} \neq \Lambda$; if $n=m$, then $2^{n} \epsilon \phi\left(2^{m}\right)$; if $n \in \phi\left(2^{m}\right)$, then $2^{n} \in \phi\left(2^{m}\right)$. 
(2) $\phi\left(2^{m}\right) \subseteq \phi(m)-\{m\}$ : by $4.6,2^{m} \in \phi(m)-\{m\}$; assume $n \in \phi(m)-$ $\{m\}$ and $2^{n} \neq \Lambda$. So $2^{n} \in \phi(m) ;$ by $6.4, m \leq n$; by 4.6 and $4.8, m<2^{m} \leq$ $2^{n}$, so $2^{n} \in \phi(m)-\{m\}$.

6.7 If $2^{m} \neq \Lambda$, then $N c(\phi(m))=N c\left(\phi\left(2^{m}\right)\right)+1(6.5$ and 6.6)

6.8 If $2^{m}=\Lambda$, then $N c(\phi(T(m)))=2$ or 3 .

Proof: By the hypothesis and 4.5, $N c(U S C(V))<m$; so by 5.2, 5.5, $T(m) \geq T(N c(U S C(V)))=N c\left(U S C^{2}(V)\right)$. So by 4.8 and $4.4,2^{T(m)} \geq$ $2^{N_{c}\left(U S C^{2}(V)\right)}=N c(U S C(V))$. If $2^{T(m)}>N c(U S C(V))$, then $\phi(T(m))=$ $\left\{T(m), 2^{T(m)}\right\}$. If $2^{T(m)}=N c(U S C(V))$, then by $4.32^{N c(U S C(V))}=N c(V)$ and $\phi(T(m))=\{T(m), N c(U S C(V)), N c(V)\}$.

7.1 If $\phi(T(m))$ is finite, so is $\phi(m)$.

Proof by induction on $N c(\phi T(m))$ : If $2^{m}=\Lambda$, then $\phi(m)=\{m\}$. If $2^{m} \neq \Lambda$, then by $5.8,5.9,6.7, N c(\phi(T(m)))=N c\left(\phi\left(2^{T(m)}\right)\right)+1=N c$ $\left(\phi\left(T\left(2^{m}\right)\right)\right)+1$; so by $3.6, N c\left(\phi\left(T\left(2^{m}\right)\right)\right)<N c(\phi(T(m)))$. If $\phi\left(2^{m}\right)$ is finite, so is $\phi(m)$ by 6.7 and 3.3 .

7.2 If $\phi(m)$ is finite, so is $\phi(T(m))$ and $N c(\phi(T(m)))=T(N c(\phi(m)))+$ $k$, where $k=1$ or $k=2$.

Proof by induction on $N c(\phi(m))$ : (We have achieved stratification by introducing a " $T$ " on the right-hand side.) Assume $N c(\phi(m))=1$; so $\phi(m)=\{m\}, 2^{m}=\Lambda$; so by $6.8, N c(\phi(T(m)))=2$ or 3 , by $5.2,2=$ $T(1)+1,3=T(1)+2$. Assume $N c(\phi(m))>1$; so $2^{m} \neq \Lambda$ and $N c$ $(\phi(m))=N c\left(\phi\left(2^{m}\right)\right)+1$. By 3.6, $N c\left(\phi\left(2^{m}\right)\right)<N c(\phi(m))$; by 5.2, 5.3, and 6.6, $N c(\phi(T(m)))=N c\left(\phi\left(2^{T(m)}\right)\right)+1=N c\left(\phi\left(T\left(2^{m}\right)\right)\right)+1=$ $T\left(N c\left(\phi\left(2^{m}\right)\right)\right)+k+1=T\left(N c\left(\phi\left(2^{m}\right)\right)+1\right)+k=T(N c(\phi(m)))+k$, where $k=1$ or 2 .

7.3 There is a cardinal number $m$ such that $\phi(m)$ is finite and $T(m)=m$.

Proof: Let $c$ be the set of cardinal numbers $n$ such that $\phi(n)$ is finite. By 6.3, $c$ is not the null set. Let $m$ be the smallest cardinal number in $c$; so $\phi(m)$ is finite. By 7.2, $\phi(T(m))$ is finite, so $m \leq T(m)$. By 5.6 , there is a cardinal number $p$ such that $m=T(p) ; T(p) \leq T(T(p))$ and by 5.5 , $p \leq T(p)$. By 7.1, $\phi(p)$ is finite, so $p=T(p), m=T(m)$.

7.4 There is a finite cardinal number $n$ such that $n=T(n)+1$ or $n=T(n)+2$.

Proof: Choose $m$ such that $\phi(m)$ is finite and $T(m)=m$ and let $n=$ $N c(\phi(m))$. By 7.2, $n=N c(\phi(T(m)))=T(N c(\phi(m)))+k=T(n)+k$, where $k=1$ or 2 .

7.5 Contradiction: 5.4 and $7.4 .^{4}$

8.1 Generalized continuum hypothesis in "New Foundations": If $m$, $2^{m}, n$ are cardinal numbers, $m$ not finite and $m \leq n \leq 2^{m}$, then either $m=n$ or $n=2^{m}$. The generalized continuum hypothesis does not hold in "NF."' The proof is by proving the theorem of Lindenbaum and Tarski in "NF" according to which the axiom of choice is a consequence of the generalized continuum hypothesis. 
${ }^{1}$ Quine, W. V., "New Foundations for Mathematical Logic," Am. Math. Monthly, 44, 70-80 (1937).

2 The axiom of infinity has been proved in a paper submitted to the Journal of Symbolic Logic; the constant use of cardinal number in the present note goes back to the referee of that paper.

${ }^{3}$ Rosser, J. B., Logic for Mathematicians, McGraw-Hill Book Company, Inc., New York, 1953. References to this book will be of the form "( $R$ 256)," where the number indicates the page.

4 By a slight modification, we can prove the following theorem (without the axiom of choice): If $m=N c(a)=N c(S C(a))$, then there is a cardinal number $n$ such that neither $n \leq T(m)$ nor $T(m) \leq n$. A finite set is therefore not equivalent to its power set; this has been proved in the paper mentioned in reference 2 .

5 This has been proved in the paper mentioned in reference 2 with the axiom of choice.

\title{
NUCLEAR REACTIONS WITH ENERGETIC NITROGEN IONS
}

\author{
By H. L. Reynolds, D. W. Scotr, AND A. ZuCker
}

\section{OAK Ridge National Laboratory}

Communicated by Gregory Breit, June 29, 1953

1. Introduction.-The possibility of obtaining information concerning the nucleus with accelerated heavy ions was discussed recently by Breit, Hull, and Gluckstern. ${ }^{1}$ Several sources of energetic heavy nuclei have been reported. Sextuply-charged carbon ions have been accelerated in the 60 -inch cyclotron ${ }^{2}$ at Berkeley and in the 170 -inch synchrocyclotron ${ }^{3}$ at the University of Chicago to energies of $120 \mathrm{Mev}$ and $1000 \mathrm{Mev}$, respectively. The Berkeley group has reported several reactions produced by carbon ions, including some reactions resulting in the production of californium. ${ }^{4}$ Recently, Miller ${ }^{5}$ investigated nuclear reactions occurring in emulsions exposed to the external carbon beam of the 60 -inch cyclotron. Heavy nuclei have been observed in nuclear emulsions exposed to cosmic rays at high altitudes, but, due to the high energies and low fluxes of these cosmic-ray particles, ${ }^{6}$ they do not lend themselves conveniently to a study of nuclear properties at low energies.

The experiments reported here utilized triply-charged nitrogen ions accelerated in the ORNL 63-inch cyclotron to an $\mathrm{H} \rho$ corresponding to an energy of approximately $25 \mathrm{Mev}$. They represent an extension of the activation experiments of Wyly and Zucker ${ }^{7}$ in which radioactive products from nitrogen bombarded targets are detected and identified mainly from their half-lives. The choice of nitrogen as the accelerated particle was based on the following considerations: (1) the magnetic field available to the resources of the program was limited to an $\mathrm{H} \rho$ of $3.75 \times 10^{5}$ oerstedinches; in order that the accelerated particles penetrate the coulomb 